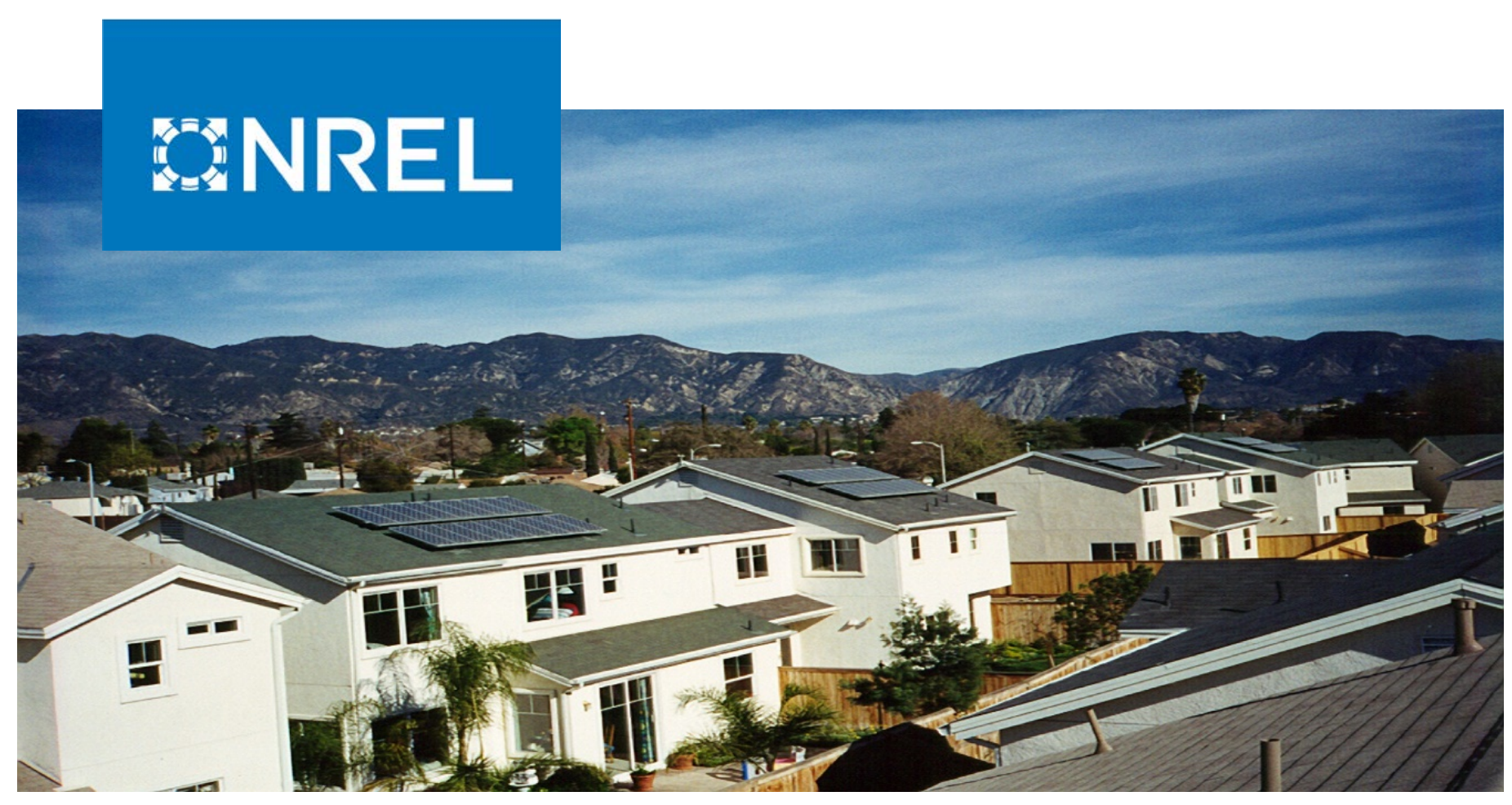

\title{
Coordinating Distributed Energy Resources for Grid Services: A Case Study of Pacific Gas and Electric
}

Kristen Ardani, Eric O'Shaughnessy, and Paul Schwabe

National Renewable Energy Laboratory

NREL is a national laboratory of the U.S. Department of Energy

Office of Energy Efficiency \& Renewable Energy

Operated by the Alliance for Sustainable Energy, LLC

This report is available at no cost from the National Renewable Energy Laboratory (NREL) at www.nrel.gov/publications.

\section{Technical Report}

NREL/TP-7A40-72108

November 2018 


\section{GNREL}

Coordinating Distributed Energy Resources for Grid Services: A Case Study of Pacific Gas and Electric

Kristen Ardani, Eric O'Shaughnessy, and Paul Schwabe National Renewable Energy Laboratory

\section{Suggested Citation}

Ardani, Kristen, Eric O'Shaughnessy, and Paul Schwabe. 2018. Coordinating Distributed Energy Resources for Grid Services: A Case Study of Pacific Gas and Electric. Golden, CO: National Renewable Energy Laboratory. NREL/TP-7A40-72108.

https://www.nrel.gov/docs/fy19osti/72108.pdf.

NREL is a national laboratory of the U.S. Department of Energy Office of Energy Efficiency \& Renewable Energy Operated by the Alliance for Sustainable Energy, LLC

This report is available at no cost from the National Renewable Energy Laboratory (NREL) at www.nrel.gov/publications.

Contract No. DE-AC36-08GO28308
Technical Report NREL/TP-7A40-72108 November 2018

National Renewable Energy Laboratory 15013 Denver West Parkway Golden, CO 80401

303-275-3000 • www.nrel.gov 


\section{NOTICE}

This work was authored by the National Renewable Energy Laboratory, operated by Alliance for Sustainable Energy, LLC, for the U.S. Department of Energy (DOE) under Contract No. DE-AC36-08G028308. Funding provided by U.S. Department of Energy Office of Energy Efficiency and Renewable Energy Solar Energy Technologies Office. The views expressed herein do not necessarily represent the views of the DOE or the U.S. Government.

This report is available at no cost from the National Renewable Energy Laboratory (NREL) at www.nrel.gov/publications.

U.S. Department of Energy (DOE) reports produced after 1991 and a growing number of pre-1991 documents are available free via www.OSTI.gov.

Cover Photo by Ed Hancock, NREL 09759.

NREL prints on paper that contains recycled content. 


\section{Acknowledgments}

This work was funded by the U.S. Department of Energy's (DOE's) Solar Energy Technologies Office. The authors would like to thank Pacific Gas and Electric (PG\&E), Tesla, General Electric, and ENGIE Storage for contributing their expertise to this study. We also would like to thank the following individuals and organizations for their review of this work: Michael Coddington (National Renewable Energy Laboratory [NREL]), Bryan Ho (ENGIE), Robert Margolis (NREL), Garrett Nilsen (DOE's Solar Energy Technologies Office), Alex Portilla (PG\&E), and Fulin Zhuang. Finally, we would like to thank Jarett Zuboy for editorial support. 


\section{List of Acronyms}

$\begin{array}{ll}\text { ADMS } & \text { advanced distribution management system(s) } \\ \text { BTM } & \text { behind-the-meter } \\ \text { C\&I } & \text { commercial and industrial } \\ \text { CAISO } & \text { California Independent System Operator } \\ \text { CPUC } & \text { California Public Utilities Commission } \\ \text { DER } & \text { distributed energy resource } \\ \text { DERMS } & \text { distributed energy resource management system(s) } \\ \text { EPIC } & \text { Electric Program Investment Charge } \\ \text { IEEE } & \text { Institute of Electrical and Electronics Engineers } \\ \text { kVAR } & \text { kilovolt ampere reactive } \\ \text { kW } & \text { kilowatt } \\ \text { kWh } & \text { kilowatt-hour } \\ \text { MUA } & \text { multiple-use applications } \\ \text { MVA } & \text { mega volt amp } \\ \text { MW } & \text { megawatt } \\ \text { MWh } & \text { megawatt-hour } \\ \text { PG\&E } & \text { Pacific Gas and Electric } \\ \text { PV } & \text { photovoltaic(s) } \\ \text { RFO } & \text { request for offer } \\ \text { RFP } & \text { request for proposals } \\ \text { SCADA } & \text { supervisory control and data acquisition } \\ \text { TCM } & \text { technology capability maturity } \\ \text { TD\&D } & \text { technology demonstration and deployment } \\ \text { VCM } & \text { vendor capability maturity }\end{array}$




\section{Executive Summary}

Pacific Gas and Electric (PG\&E) hosts more distributed solar photovoltaic (PV) systems than any other utility in the United States, and it expects hundreds of thousands of new PV systems, numerous electric vehicles, and more than 400 megawatts (MW) of behind-the-meter distributed energy storage by 2030. To prepare for the grid of the future, PG\&E is exploring ways to coordinate distributed energy resources (DERs) to increase grid flexibility, reliability, and hosting capacity. This case study explores PG\&E's demonstration of a software approach to coordinating diverse DERs - known as a DER management system (DERMS) - in a way that maintains proper grid operation while enabling DERs to provide grid services.

The PG\&E DERMS technology demonstration implemented a DERMS developed by GE Grid Solutions for managing 124 kilowatts $(\mathrm{kW})$ of residential PV coupled with $66 \mathrm{~kW}$ (264 kilowatthours $[\mathrm{kWh}])$ of residential storage at 27 homes, $360 \mathrm{~kW}(720 \mathrm{kWh})$ of commercial storage at three commercial locations, and a 4-MW (28-megawatt-hour [MWh]) PG\&E-owned utility-scale battery (Figure ES-1). PG\&E partnered with Tesla to coordinate the residential DERs and ENGIE Storage to coordinate the commercial DERs.

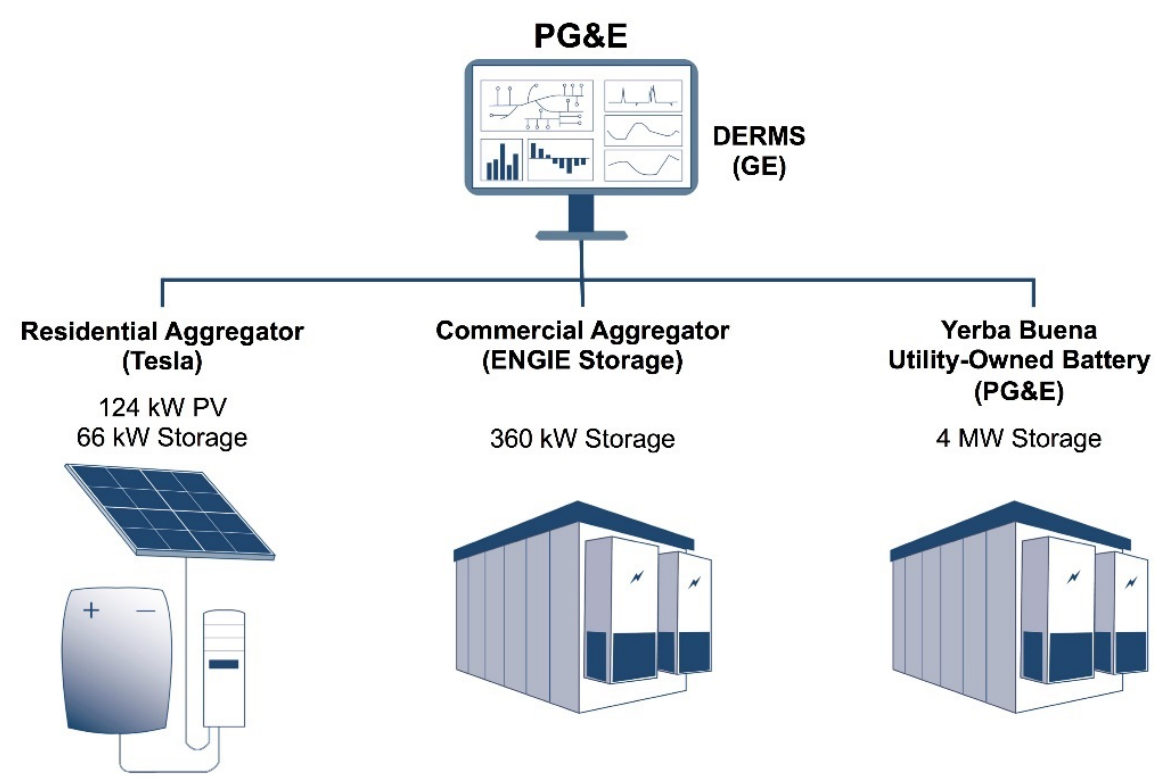

Figure ES-1. PG\&E DERMS demonstration asset fleet

The PG\&E DERMS demonstration is among the first field validations of DERMS core capabilities in the United States. Among several key outcomes, it showed that a DERMS can be used to coordinate DERs, leverage DERs to mitigate capacity constraints and voltage violations, and provide distribution grid services while potentially enabling DER resources to bid into wholesale markets. This case study analyzes the PG\&E DERMS demonstration project to identify outcomes, lessons learned, challenges, and possible next steps. Specifically, DERMS performance is evaluated across seven use cases:

- Provide situational awareness

- Manage equipment capacity constraints and reverse power flow 
- Mitigate voltage issues with real-power output

- Mitigate voltage issues with reactive power

- Enable economic dispatch of distributed generation and energy storage

- Provide operational flexibility

- Enable limited multiple-use applications of DERs

The DERMS demonstration produced positive outcomes for each case while revealing various specific issues and areas for improvement. The following are highlights of the challenges identified, along with next steps required for integrating DERMS into utility operational systems at scale.

Scalability - Although the DERMS demonstration successfully coordinated several aggregated DER resources, future DERMS may need to coordinate DER fleets with thousands of systems and much higher capacities. Requirements for operating the future high-DER-penetration grid may include utility investment in foundational capabilities and systems (e.g., planning tools and cybersecurity measures), coordination of DERs with traditional distribution operations equipment, improved telemetry for increasing visibility into the operational status and availability of DERs, and a scalable and interoperable DER aggregation platform that enables real-time, two-way secure communication between DERs/DER aggregations and utility systems.

Standardization-Aggregator competition could spur innovation and reduce costs, but markets with many aggregators, software products, protocols, and standards may present a challenge to utilities. More standardization may be necessary to ensure that utility requests are interpreted consistently across aggregators, ensure a common understanding of fundamental concepts such as flexibility, and measure key operating metrics to assess aggregator performance.

Targeted DER deployment - The challenges PG\&E encountered in recruiting demonstration participants may foreshadow broader challenges related to targeted DER deployment. Ideally, DERs would be sited where they can provide the greatest value to the grid, but siting may depend on customer acquisition and the interactions of customers with third-party aggregators, which may not yield an optimal site portfolio from a grid perspective.

Valuation - The demonstration did not quantify the value of DER-provided services or the costs of avoided violations. Further research is required to clarify the valuation of distribution services.

Market design - The demonstration showed that DERs could be coordinated through an askbid-commit market structure, but it did not address critical compensation, settlement, market design, or market rules issues. The ask-bid-commit market structure may not be viable until key market-design elements are determined. In the near term, simpler dispatch methods, such as direct control or signaling, will likely demonstrate greater ease of implementation.

Operational flexibility - The DERMS demonstration did not incorporate DERs into optimal switching plans. Future projects could group DERs into multiple aggregations, each capable of operating autonomously from the main aggregation, in the case of a switching operation. 
Human capital - The future of the electric grid may rely increasingly on strategic partnerships between utilities, technology vendors, and aggregators that work toward maximizing the value of DERs to the grid. With the necessary ongoing commitment and investments, projects like the PG\&E DERMS demonstration can play an important role in achieving this goal. 


\section{Table of Contents}

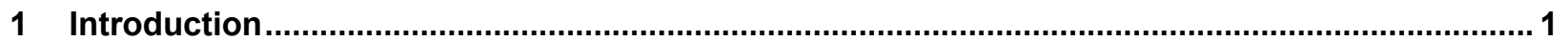

2 PG\&E DERMS Demonstration: Objectives, Development, and Implementation ......................... 3

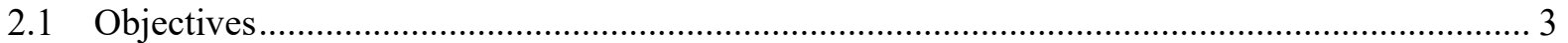

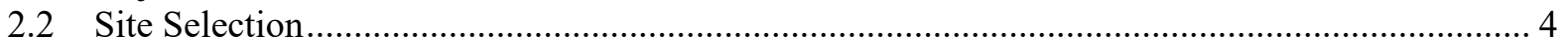

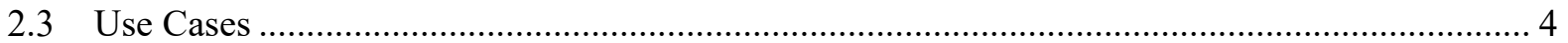

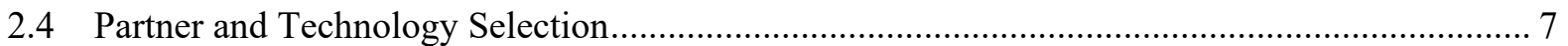

2.5 Demonstration Implementation Partner Responsibilities and Assets ................................... 8

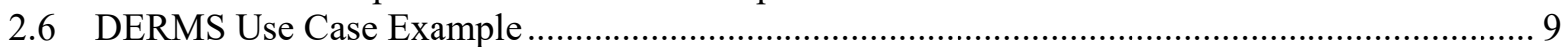

2.7 DERMS Integration with Third-Party Aggregated DERs and Utility-Owned DERs ............... 11

3 Key Outcomes and Lessons Learned .............................................................................. 13

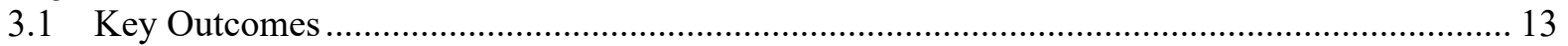

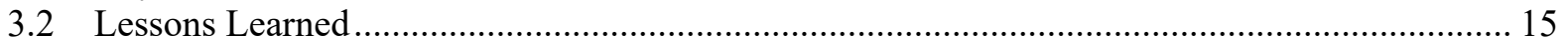

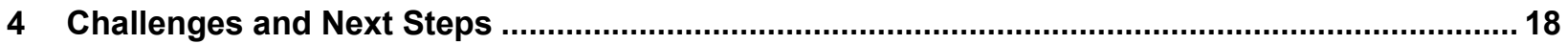

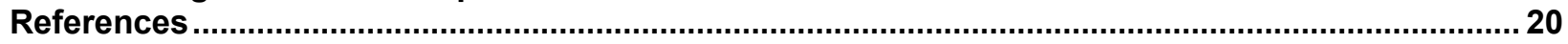

Appendix: The Electric Program Investment Charge (EPIC) Program ........................................... 21 


\section{List of Figures}

Figure ES-1. PG\&E DERMS demonstration asset fleet and partner responsibilities for assets ..................vi

Figure 1. DER aggregation demonstration programs (program start years in parentheses) ........................ 1

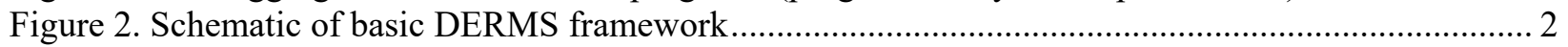

Figure 3. DERMS demonstration location and six aggregation nodes ................................................... 4

Figure 4. PG\&E DERMS demonstration asset fleet and partner responsibilities for assets ....................... 9

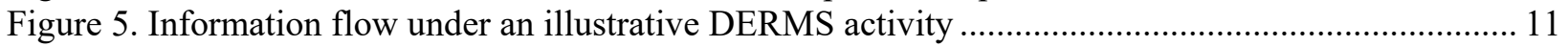

Figure 6. Functional blocks and logic of interactions between subsystems used in the demonstration ..... 12

Figure 7. Static illustration of PG\&E DERMS demonstration software interface (PG\&E 2017) ............. 14

Figure 8. Forecasted DERMS-enabled overload mitigation example................................................... 15

Figure 9. Subset of PG\&E DER-related TD\&D portfolio (PG\&E 2017) ............................................... 22

\section{List of Tables}

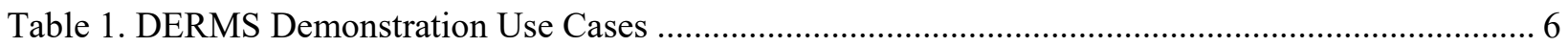

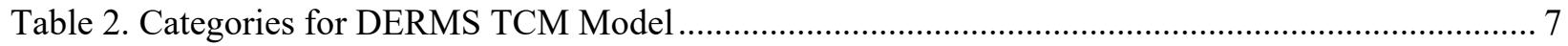

Table 3. PG\&E DERMS Demonstration Project Partner Responsibilities ................................................ 9

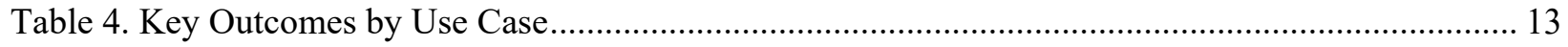

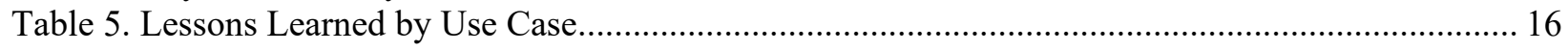




\section{Introduction}

Pacific Gas and Electric (PG\&E) is one of many U.S. utilities working to integrate increasing penetrations of distributed energy resources (DERs) such as solar photovoltaics (PV), electric vehicles, load control, and behind-the-meter (BTM) energy storage (Figure 1). The PG\&E distribution system hosts more distributed PV capacity than any other system in the United States, with more than 380,000 distributed PV systems installed as of August 2018 (PG\&E 2018). PG\&E expects accelerated adoption of PV and other DERs, including hundreds of thousands of new PV systems, numerous electric vehicles, and more than 400 megawatts (MW) of BTM energy storage by 2030 (PG\&E 2017).

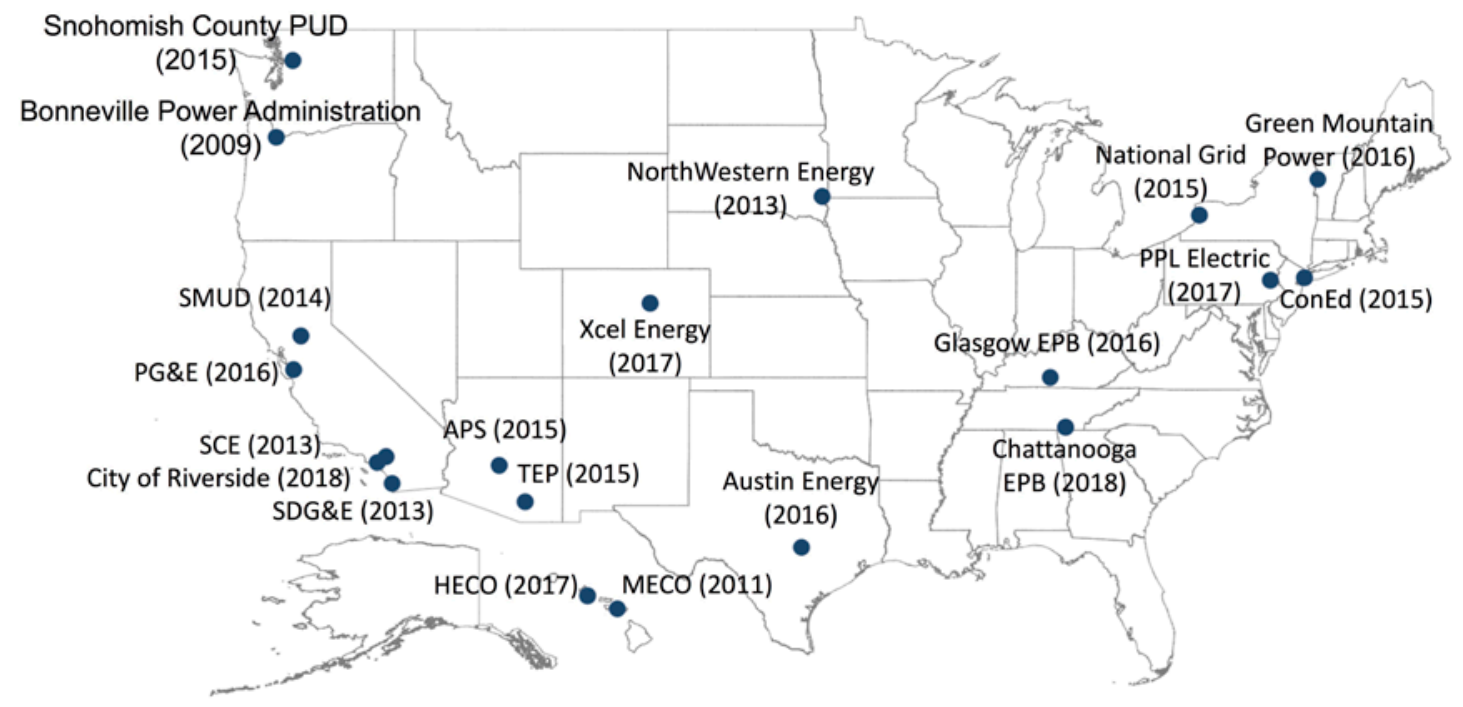

Figure 1. DER aggregation demonstration programs (program start years in parentheses)

DERs have potential to provide valuable services to retail electricity customers, distribution grids, and the bulk electric system - when the DERs are present in sufficient quantities and in suitable locations, available when needed for specific durations, and more cost-effective than other approaches to providing the services. However, DER growth also entails grid-integration challenges, including managing capacity constraints and equipment overload, managing reverse power flow, mitigating voltage and frequency issues, and achieving adequate DER visibility so DERs can be integrated safely and reliably into distribution system operations under normal or abnormal grid configurations. ${ }^{1}$

DER management systems (DERMS) are an emerging solution for aggregating and integrating high levels of DER penetration and potentially enabling DERs to increase grid reliability, flexibility, and hosting capacity (Braff et al. 2016). Although the definition of the DERMS technology continues to evolve, in general it is a system that provides "the ability to continuously manage diverse and dispersed DERs, both individually and in aggregate, to support multiple

\footnotetext{
${ }^{1}$ For a typical radial distribution system, abnormal grid configuration refers to the reconfiguration of grid topology to allow for more flexible operations. Examples include serving load from an adjacent feeder or substation to aid with restoration, perform maintenance, or address other grid issues. At any given time, a significant portion of the grid may be subject to an abnormal configuration, also referred to as abnormal switching.
} 
objectives related to distribution grid operations [and] end-customer value creation including market participation" (Mulherkar 2017). DERMS enable utilities to use DER operational data and capabilities to issue commands, based on market signals and grid conditions, to the DERs that mitigate DER impacts and may also provide grid services (Figure 2).

A DERMS generally fits within the framework of an advanced distribution management system (ADMS) software platform. An ADMS supports the full suite of distribution management and optimization functions, including managing the outage-restoration process and optimizing the performance of the distribution grid (Gartner 2018). A DERMS monitors and manages DERs to address the grid needs that are determined by the ADMS.

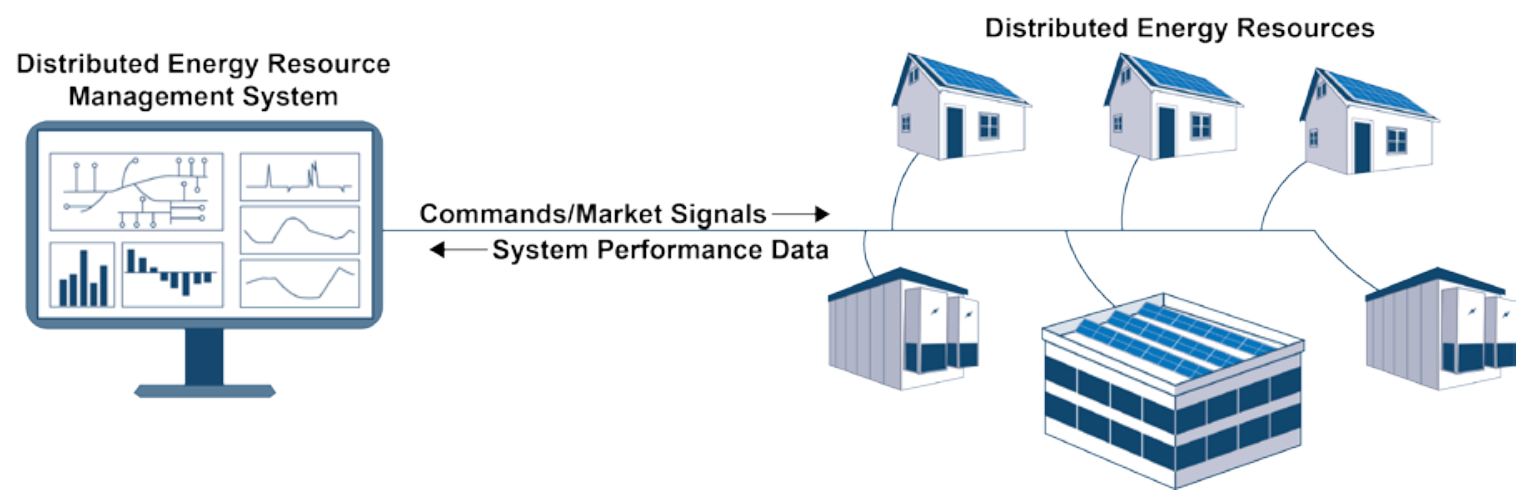

Figure 2. Schematic of basic DERMS framework

Between 2016 and 2018, PG\&E demonstrated and evaluated DERMS use in San Jose, California. This case-study report examines the design of and early lessons learned from the PG\&E DERMS demonstration. The report also describes how the DERMS demonstration allowed aggregated DERs to provide distribution services via a simulated distribution market while enabling participation in wholesale California Independent System Operator (CAISO) markets and providing BTM services, such as backup power. This case study may also be used to inform decision making at other U.S. utilities that are experiencing, or planning for, significant DER growth.

The remainder of this report is structured as follows: Section 2 describes the objectives, development, and implementation of the PG\&E DERMS demonstration. Section 3 summarizes the demonstration's key outcomes and lessons learned. Section 4 concludes by noting various challenges and next steps related to integrating DERs into utility operational systems at scale. 


\section{PG\&E DERMS Demonstration: Objectives, Development, and Implementation}

The PG\&E DERMS demonstration (EPIC 2.02: DERMS) was funded by the California Electric Program Investment Charge (EPIC) program (see the Appendix). It was implemented along with two other co-located EPIC-funded projects (EPIC 2.03A: Smart Inverter Enhanced Capabilities and EPIC 2.19C: Customer Sited and Behind-the-Meter Storage). Final reports for each of the completed PG\&E EPIC projects are available on the PG\&E website. ${ }^{2}$ This section summarizes the objectives, development, and implementation of the DERMS demonstration.

\subsection{Objectives}

According to PG\&E, the objective of the DERMS demonstration was to "demonstrate new technology to monitor and control DERs to manage system constraints and evaluate the ability to manage a 'fleet' of DERs to provide distribution grid services. The DERMS demonstration will drive learning about the people, process, and technology needed to operate the higher DER penetration grid of 2025" (PG\&E 2017). The DERMS was meant to coordinate DERs across various levels of the electric system (e.g., customer, distribution feeder, and substation). A successful DERMS would address the need for more flexible, precise, and active DER management than provided by traditional demand-side and distribution-management systems.

More specifically, the PG\&E DERMS demonstration was meant to help answer the following types of questions:

- Technical: How can the coordination of new distributed energy technologies such as smart inverters connected to PV and energy storage systems help mitigate potential DER impacts and alleviate constraints on the local distribution system via flexible operation? How can a utility DERMS enable multilayer aggregation (e.g., line segment, feeder, substation transformer), optimization, and control functionality? What are the specific technical requirements for each use case, and what boundaries and integrations are required between the DERMS and other utility systems (e.g., ADMS) to enable such use cases?

- Human capital: What types of knowledge, skills, and training are needed to deploy DERMS optimally? What level of partnership with third parties and external vendors will yield the highest-quality results when deploying DERMS? What types of knowledge, skills, and training are needed to coordinate DERs and operate DERMS optimally after those systems are deployed?

- Process: When working with third parties, what are the key criteria for partner and vendor selection when adopting and operationalizing DERMS? What stages of technology development and testing are necessary for DERMS deployment? What are the requirements regarding interaction between the utility and aggregators to coordinate third-party-operated DERs to meet utility-defined distribution grid needs? What are the

\footnotetext{
${ }^{2}$ See https://www.pge.com/en_US/about-pge/environment/what-we-are-doing/electric-program-investmentcharge/closeout-reports.page.
} 
required standards for interfaces between utility platforms and third-party DERs and software systems?

In the long term, PG\&E envisions the DERMS demonstration as an important first step toward identifying the capabilities needed to operate the "grid of the future." These capabilities will be key to advancing PG\&E's mission of safely and reliably delivering affordable, resilient, and clean energy service to its customers and communities while building the energy network of tomorrow. One component of this vision is an integrated utility platform that enables monitoring, management, and control of DERs to deliver safe, reliable, and resilient service to PG\&E's customers by leveraging the diversity of DERs.

\subsection{Site Selection}

PG\&E selected three distribution feeders connected to its Swift Substation located in San Jose, California, for the DERMs demonstration (Figure 3). These feeders serve approximately 9,500 customers and were divided into six aggregation "nodes" for the demonstration. Selection criteria included the technical characteristics of the local distribution system (e.g., existing grid sensors and DERs), local DER-adoption forecasts, and efficient use of EPIC research funds. In addition, this site leveraged geographic synergies between the DERMS demonstration and several related EPIC projects (see the Appendix). The colocation of these demonstration activities facilitated collaboration among PG\&E departments - such as Electric Operations, Information Technology, Energy Procurement, and Customer Energy Solutions - that likely will need to collaborate more as DER adoption continues to grow.

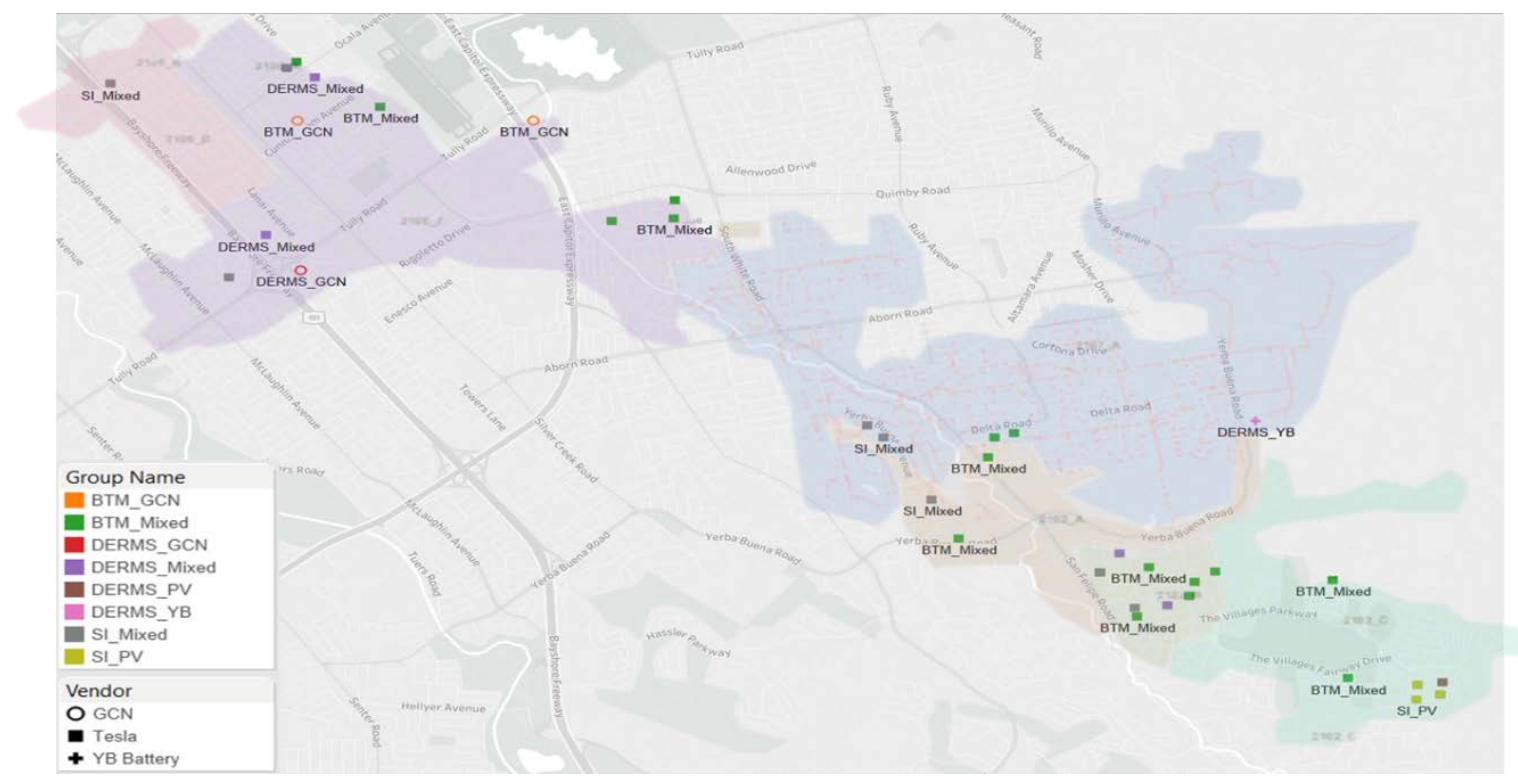

Figure 3. DERMS demonstration location and six aggregation nodes (Portilla 2017)

\subsection{Use Cases}

When identifying use cases for the DERMS demonstration, PG\&E prioritized real-time and dayahead aggregated grid-flexibility services that require a management platform with capabilities 
not commercially available among alternative management systems such as ADMS. ${ }^{3}$ PG\&E aimed to select use cases such that the DERMS demonstration augmented and integrated with other utility systems, platforms, and equipment. For example, the DERMS demonstration relied on specific ADMS applications (deployed at test scale for the demonstration) to provide situational awareness of real-time and forecasted grid conditions. Table 1 describes the seven DERMS demonstration use cases.

\footnotetext{
${ }^{3}$ These capabilities were not available when the demonstration project was designed and DERMS software was procured. Advances in ADMS since then may provide some of the targeted functionality, but not yet in an integrated and mature solution as would be required to scale up all the capabilities explored in this demo.
} 
Table 1. DERMS Demonstration Use Cases

\begin{tabular}{|c|c|c|}
\hline Use Case & Description & PG\&E Demonstration Application \\
\hline $\begin{array}{l}\text { Provide situational } \\
\text { awareness }\end{array}$ & $\begin{array}{l}\text { Visualize actual and forecasted } \\
\text { DER-related grid conditions in } \\
\text { real time: DER generation, } \\
\text { customer load, net load (customer } \\
\text { load less DER generation), and } \\
\text { DER flexibility. }\end{array}$ & $\begin{array}{l}\text { The demonstration included ADMS } \\
\text { applications with DER real-time and forecast } \\
\text { modeling capabilities, as well as adequate } \\
\text { telemetry and monitoring capabilities to } \\
\text { field-verify application performance. }\end{array}$ \\
\hline $\begin{array}{l}\text { Manage equipment } \\
\text { capacity constraints } \\
\text { and reverse power } \\
\text { flow }\end{array}$ & $\begin{array}{l}\text { Control DERs to mitigate overload } \\
\text { issues dynamically through } \\
\text { operational strategies (e.g., } \\
\text { selective charging/discharging of } \\
\text { dispatchable assets and/or power } \\
\text { curtailment of smart inverter } \\
\text { output). }\end{array}$ & $\begin{array}{l}\text { The demonstration tested whether a } \\
\text { DERMS can coordinate DERs to mitigate } \\
\text { real-time or forecasted overloads and } \\
\text { reverse-flow power conditions, as well as } \\
\text { whether a portfolio of aggregated DERs can } \\
\text { be managed to serve as a non-wires } \\
\text { alternative to defer equipment capacity } \\
\text { upgrades. }\end{array}$ \\
\hline $\begin{array}{l}\text { Mitigate voltage } \\
\text { issues with real- } \\
\text { power output }\end{array}$ & $\begin{array}{l}\text { Leverage DER flexibility to } \\
\text { resolve an existing voltage issue } \\
\text { by altering real-power output } \\
\text { (e.g., selective charging/ } \\
\text { discharging of dispatchable } \\
\text { assets and/or power curtailment } \\
\text { of smart inverter controlled DER). }\end{array}$ & $\begin{array}{l}\text { The demonstration tested whether a } \\
\text { DERMS can coordinate DERs to mitigate } \\
\text { real-time or forecasted voltage violations by } \\
\text { dispatching real power. }\end{array}$ \\
\hline $\begin{array}{l}\text { Mitigate voltage } \\
\text { issues with reactive } \\
\text { power }\end{array}$ & $\begin{array}{l}\text { Leverage smart inverter settings } \\
\text { and dynamic controls to generate } \\
\text { reactive power to support voltage } \\
\text { stability, e.g., kilovolt ampere } \\
\text { reactive (kVAR) dispatch and/or } \\
\text { mode control of smart inverters to } \\
\text { set power factor. }\end{array}$ & $\begin{array}{l}\text { The demonstration tested whether a } \\
\text { DERMS can coordinate DERs to mitigate } \\
\text { real-time voltage violations by dispatching } \\
\text { reactive power. }\end{array}$ \\
\hline $\begin{array}{l}\text { Economic dispatch } \\
\text { of distributed } \\
\text { generation and } \\
\text { energy storage }\end{array}$ & $\begin{array}{l}\text { Dispatch DERs based on } \\
\text { economic factors such as cost or } \\
\text { external pricing signals. }\end{array}$ & $\begin{array}{l}\text { The demonstration employed an "ask-bid- } \\
\text { commit" simulated market structure to } \\
\text { perform least-cost dispatch of DERs on a } \\
\text { day-ahead and hourly basis. }\end{array}$ \\
\hline $\begin{array}{l}\text { Operational } \\
\text { flexibility }\end{array}$ & $\begin{array}{l}\text { Demonstrate that DERMS can be } \\
\text { used to develop forecasts and } \\
\text { optimizations during abnormal } \\
\text { switching configurations. }{ }^{4}\end{array}$ & $\begin{array}{l}\text { The demonstration tested whether a } \\
\text { DERMS can mitigate forecasted capacity } \\
\text { and voltage issues by coordinating DERs } \\
\text { during abnormal switching configurations. }\end{array}$ \\
\hline $\begin{array}{l}\text { Enable multiple-use } \\
\text { applications (MUAs) } \\
\text { of DERs }\end{array}$ & $\begin{array}{l}\text { Enable DERs to provide value to } \\
\text { both the distribution grid and the } \\
\text { wholesale market (i.e., provide } \\
\text { value stacking). }\end{array}$ & $\begin{array}{l}\text { The demonstration tested whether a } \\
\text { DERMS can coordinate BTM and front-of- } \\
\text { the-meter batteries to provide distribution } \\
\text { grid services while also bidding into } \\
\text { wholesale energy markets in a limited set of } \\
\text { scenarios }\end{array}$ \\
\hline
\end{tabular}

\footnotetext{
${ }^{4}$ At any given time, a significant portion of the grid may be subject to an abnormal configuration, also referred to as abnormal switching.
} 


\subsection{Partner and Technology Selection}

PG\&E selected an external partner and DERMS technology for the demonstration via a competitive sourcing process with a robust scoring system and evaluation criteria. A primary goal was to create a minimum viable product to facilitate cost-effective learning about future DERMS requirements. PG\&E structured the request for proposals (RFP) and demonstration to inform future vendor selection and DERMS functionality requirements, rather than procuring a long-term DERMS for use beyond this project.

To evaluate proposals across numerous DERMS technologies, PG\&E used a technology capability maturity (TCM) model that measured 25 dimensions of DERMS capability across six categories (Table 2). This method was chosen instead of a traditional requirements-based RFP because the technology at the time was nascent (and still continues to evolve rapidly). The TCM model uses a six-point scale (0 to 5) to benchmark against PG\&E's desired and minimal DERMS functionality requirements. This approach facilitates the objective evaluation of technology risk relative to the project's stated goals and is well-suited for evaluating nascent technology.

Table 2. Categories for DERMS TCM Model

\begin{tabular}{|c|c|}
\hline Category & DERMS Capability \\
\hline Optimization & $\begin{array}{l}\text { Automatically optimize DER dispatch across multivariate engineering, economic, } \\
\text { contractual, and regulatory parameters. Simultaneously process local and system- } \\
\text { level optimizations. }\end{array}$ \\
\hline $\begin{array}{l}\text { Measurement, } \\
\text { Analysis, \& } \\
\text { Reconciliation }\end{array}$ & $\begin{array}{l}\text { Measure device output or impact on a given configuration, analyze specific data } \\
\text { (leading to potential optimization solutions), and forecast the future state of an asset } \\
\text { (or prices) to influence selection, use, and control of assets and reconciliation of } \\
\text { events in the context of electrical, economic, and/or contractual obligations. }\end{array}$ \\
\hline $\begin{array}{l}\text { Life-Cycle } \\
\text { Management }\end{array}$ & $\begin{array}{l}\text { Enable assets to auto-register, manage listed constraints, remotely test and } \\
\text { characterize assets, view operational parameters for individual and aggregated } \\
\text { assets, and automatically detect and alert operators of malfunctioning assets, as well } \\
\text { as remove and decommission assets that are members of the resource pool. }\end{array}$ \\
\hline $\begin{array}{l}\text { Real-Time } \\
\text { Situational } \\
\text { Intelligence }\end{array}$ & $\begin{array}{l}\text { Receive static and dynamic state data from a large number of assets and display such } \\
\text { data upon request, seamlessly integrate operational changes to static data into } \\
\text { analysis and reconciliation functions, receive disparate telemetry data from multiple } \\
\text { vendors' equipment/assets, and display such data upon request. }\end{array}$ \\
\hline Architecture & $\begin{array}{l}\text { Maintain the DERMS architecture as assets are acquired, developed, enhanced, and } \\
\text { retired. Optimize the demonstration by maximizing value and minimizing costs. Ensure } \\
\text { risk management through privacy, integrity, resiliency, and regulatory compliance } \\
\text { measures. Ensure that systems are reliable, available, and serviceable. }\end{array}$ \\
\hline Security & $\begin{array}{l}\text { Manage cybersecurity risk to systems, assets, data, and capabilities. Manage data } \\
\text { consistent with their relative importance to business objectives and the organization's } \\
\text { risk strategy. Implement appropriate safeguards to ensure delivery of critical } \\
\text { infrastructure services. Detect anomalous activity and cybersecurity events in a timely } \\
\text { manner, limit or contain the impacts of potential events, and implement the } \\
\text { appropriate activities to take action regarding a detected cybersecurity event. } \\
\text { Implement activities for resilience and to restore any capabilities or services that were } \\
\text { impaired as a result of a cybersecurity event. }\end{array}$ \\
\hline
\end{tabular}


In addition to the TCM model, PG\&E used a vendor capability maturity (VCM) model to evaluate each DERMS vendor. The focus of the VCM evaluation was to identify key risk areas for new technologies stemming from organizational capacity to deliver the proposed solution and the technologies needed for significant customization. Selection decisions were also based on timing to maximize benefits, such as evaluating the trade-offs between using an existing platform and creating a new platform, and a detailed risk assessment to ensure the awarded vendor and technology reduced overall delivery risks for this demonstration. The sourcing process timeline was as follows:

- DERMS vendor: On August 7, 2015, PG\&E issued a competitive RFP to select the most qualified vendor to provide a customized DERMS demonstration platform. After receiving 19 RFP responses, PG\&E announced the list of five finalists on September 22, 2015. Upon reviewing responses to a supplemental questionnaire and hosting in-person demonstrations, PG\&E selected GE Grid Solutions as the recommended DERMS vendor on November 11, 2015, prior to the official demonstration kickoff in January 2016.

- DER vendors: Near the end of January 2016, PG\&E issued a request for offer (RFO) to select residential and commercial storage vendors to deploy and aggregate DERs for the DERMS technology to coordinate. PG\&E selected Tesla (then doing business as SolarCity) and ENGIE Storage (then doing business as Green Charge Networks) to provide residential solar-plus-storage and commercial storage, respectively. ${ }^{5}$

Through the RFP and RFO processes, PG\&E gained valuable insights into nascent DERMS technology as well as PV and storage aggregation, including capabilities, pricing, and applications. Including third parties in the DERMS demonstration followed California's longterm policy and regulatory priorities of enabling third-party DER aggregators to provide distribution grid services that could result in the deferral of distribution grid investments. It also strengthened partnerships between the utility and vendors that will be critical to understanding and optimally implementing emerging grid technologies like DERs and DERMS.

\subsection{Demonstration Implementation Partner Responsibilities and Assets}

Table 3 summarizes the key responsibilities for each of the four partners in the PG\&E DERMS demonstration. Figure 4 summarizes the DERMS demonstration asset fleet and partner responsibilities for the various assets. The demonstration comprised 124 kilowatts $(\mathrm{kW})$ of residential PV coupled with $66 \mathrm{~kW}$ (264 kilowatt-hours [kWh]) of residential storage at 27 homes, $360 \mathrm{~kW}(720 \mathrm{kWh})$ of commercial storage at three commercial locations, and a 4-MW (28-megawatt-hour [MWh]) PG\&E-owned utility-scale battery.

\footnotetext{
${ }^{5}$ A third class of assets was coordinated by the DERMS in this demonstration: front-of-the-meter, utility-owned distributed energy storage. Specifically, this was the Yerba Buena 4-MW battery in the San Jose region.
} 
Table 3. PG\&E DERMS Demonstration Project Partner Responsibilities

\begin{tabular}{ll}
\hline Company & Responsibilities \\
\hline PG\&E & $\begin{array}{l}\text { Design, develop, and lead the DERMS demonstration. Coordinate all parties to } \\
\text { implement and operate the DERMS demonstration and achieve project goals. Control } \\
\text { Yerba Buena battery. }\end{array}$ \\
\hline $\begin{array}{l}\text { GE Grid } \\
\text { Solutions }\end{array}$ & $\begin{array}{l}\text { Provide a "mini ADMS" and customized DERMS software. Assume responsibility for } \\
\text { DERMS software functionality, communication protocols, and utility user training. }\end{array}$ \\
\hline $\begin{array}{l}\text { ENGIE } \\
\text { Storage }\end{array}$ & $\begin{array}{l}\text { Provide commercial and industrial (C\&I) BTM storage. Lead C\&I customer-acquisition } \\
\text { activities for demonstration. Provide grid services through control of aggregated } \\
\text { ENGIE Storage C\&I battery systems. }\end{array}$ \\
\hline Tesla & $\begin{array}{l}\text { Provide residential BTM PV and storage. Lead residential customer-acquisition } \\
\text { activities for demonstration. Provide grid services through control of aggregated Tesla } \\
\text { residential PV-plus-battery systems. }\end{array}$ \\
\hline
\end{tabular}

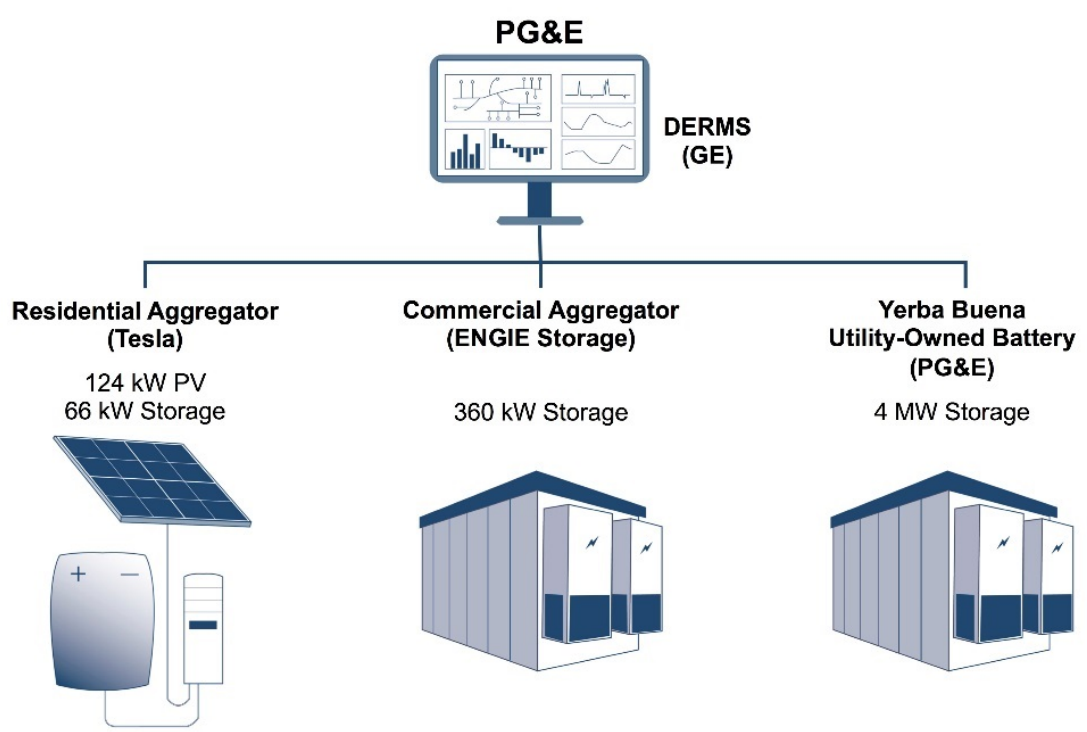

Figure 4. PG\&E DERMS demonstration asset fleet

\subsection{DERMS Use Case Example}

PG\&E demonstrated the functions and flexibility of the DERMS platform and aggregated DERs to respond to the seven use cases described in Section 2.3. The use cases varied in terms of data requirements, data sources, and sequencing of events. For example, although data for forecasted load and DER operation were commonly required across most of the use cases, the cases related to operational flexibility during switching required additional data. The specific data requirements and sequencing of events for each use case are not within the scope of this report. However, the example below (depicted in Figure 5) summarizes a DERMS demonstration scenario for managing predicted day-ahead capacity constraints using PV and storage.

Step 1 (ADMS + DERMS): Determine day-ahead flexibility needs using day-ahead load/generation forecast and real-time telemetry from supervisory control and data acquisition (SCADA) devices to forecast load flow calculations at all points along the demonstration 
distribution feeders. The following day-ahead predictions are generated on an ongoing basis (1hour recalculations):

- Real-time and forecasted grid conditions (current and voltage at specific locations on each distribution circuit) for next 36 hours at all points along the feeders

- Potential overloads at a substation transformer bank, based on transformer mega volt amp limits, conductor capacity limits, and switching device capacity limits

- Potential substation back-feed overload based on reverse-flow limits on equipment.

Step 2 (DERMS): The DERMS automatically issues a flexibility request based on constraints calculated in Step 1, which is referred to as an "ask." This step, along with the others, is autonomous and will require minimal human intervention.

Step 3 (aggregator platforms): The aggregators receive the "ask" and provide an offer of flexibility service based on the capability of DERs under control, known as a "bid." The aggregators post bids to the DERMS platform.

Step 4 (DERMS): Upon receipt of a set of kW flexibility bids, the DERMS performs a costminimization optimization to determine a day-ahead mitigation plan that includes scheduling for grid battery charging/discharging and aggregator bid activation subject to grid operating constraints.

Step 5 (PG\&E demonstration team): The DERMS operator reviews and then accepts or rejects the day-ahead mitigation plan. If the day-ahead plan is accepted, the DERMS operator dispatches the plan to the aggregators.

Step 6 (DERMS): The DERMS software posts the winning awards to the software interface, which is referred to as the "commit."

Step 7 (DERMS and aggregator platforms): At the appointed time, the aggregators provide the requested flexibility service using their respective controllable DERs, and they report delivered flexibility to the DERMS. The DERMS produces a record of the ask, bid, and commit process (e.g., optimization inputs, outputs, DERMS engineer approvals/rejections, and aggregator bids/acknowledgments of aggregation node commitments).

Step 8 (aggregator platforms): The aggregators generate an end-of-day performance report. 


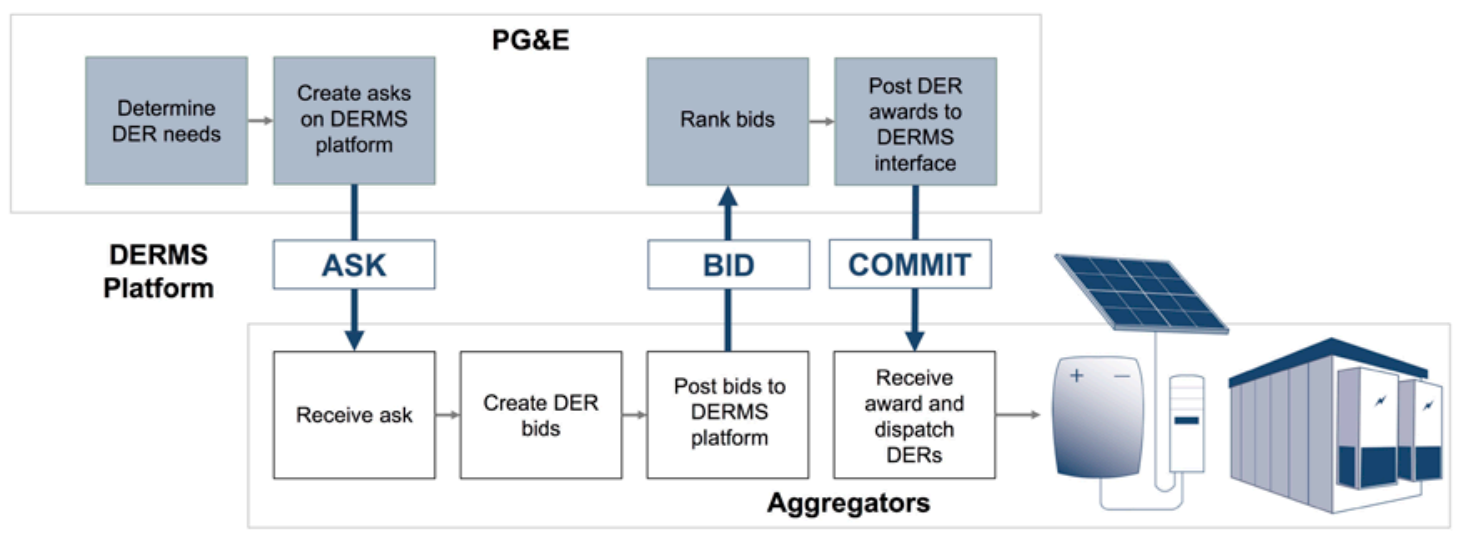

Figure 5. Information flow under an illustrative DERMS activity

\subsection{DERMS Integration with Third-Party Aggregated DERs and Utility- Owned DERs}

To integrate the DERMS with third-party aggregated DERs, PG\&E required communication pathways and protocols that enabled issuance of signals to and receipt of information from the aggregated set of DERs. For the DERMS demonstration, PG\&E tested integration using secure internet for a utility-to-aggregator software platform and relied on the aggregator for communication with each individual DER. ${ }^{6}$

PG\&E identified two protocols with built-in support for DERs: IEEE 2030.5 and OpenADR $2.0 \mathrm{~b}$. However, at the time, neither protocol could support all functionality required by the use cases without custom extensions. PG\&E ultimately chose the IEEE 2030.5 protocol because of the protocol's existing smart inverter capability, project participant familiarity with the protocol, and the California Smart Inverter Working Group's recommendation of IEEE 2030.5 as the default protocol for remote control of DERs controlled by smart inverters (CEC and CPUC 2015). Further, the OpenADR protocol was incompatible, at the time, with reactive power support.

The inability of existing standard protocols to meet all grid service use cases highlights the need for further development in this emerging area. PG\&E will work with the project partners to incorporate the custom extensions developed for this project into the IEEE 2030.5 standard where appropriate. PG\&E recognizes that, in the long term, a utility DERMS likely must be able to integrate across multiple protocols depending on the asset and DER aggregation program design.

Integration between the DERMS and the utility-owned battery storage was conducted via a combination of manual operations and real-time telemetry. The telemetry employed PG\&E's existing SCADA system using the standard DNP3 protocol.

\footnotetext{
${ }^{6}$ PG\&E interacted with the aggregators via a single software interface, and the aggregators were then responsible for transmitting the commands to individual devices.
} 
Figure 6 illustrates the functional blocks and logic of the interactions between subsystems used in the demonstration.

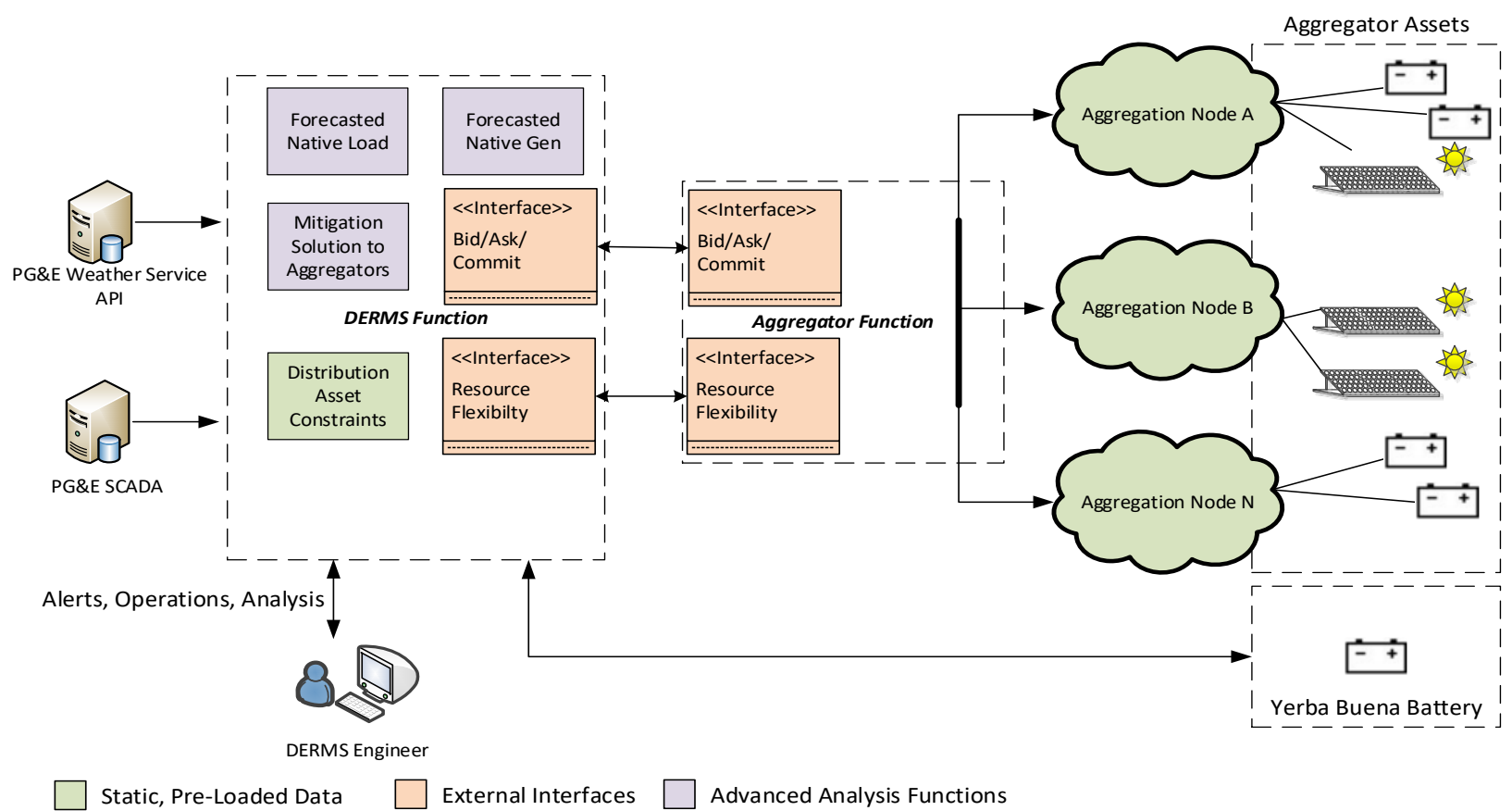

Figure 6. Functional blocks and logic of interactions between subsystems used in the demonstration (Portilla 2017) 


\section{Key Outcomes and Lessons Learned}

This section summarizes qualitative early findings and lessons learned from the PG\&E demonstration. Quantitative results will be summarized in the EPIC 2.02 final report later in 2018.

\subsection{Key Outcomes}

PG\&E's DERMS demonstration - among the first field tests of core DERMS capabilities in the United States_-found the following:

- A utility DERMS together with an ADMS can coordinate DERs to identify and mitigate real-time and future capacity constraints, voltage violations, and other potential problems.

- The combination of an ADMS and a DERMS may offer the capability needed to coordinate DER participation in wholesale markets and to avoid problems that might arise on the distribution system.

Table 4 summarizes key outcomes for each of the seven use cases.

Table 4. Key Outcomes by Use Case

\begin{tabular}{ll}
\hline Use Case & Key Outcomes \\
\hline $\begin{array}{l}\text { Provide situational } \\
\text { awareness with DERs }\end{array}$ & $\begin{array}{l}\text { The demonstration modeled real-time and forecasted conditions } \\
\text { on the distribution system. It also incorporated load and PV } \\
\text { generation forecasts and DER schedules to calculate and display } \\
\text { real-time and anticipated hidden loads and voltage across the } \\
\text { demonstration feeders. }\end{array}$ \\
\hline $\begin{array}{l}\text { Manage equipment } \\
\text { capacity constraints and } \\
\text { reverse power flow }\end{array}$ & $\begin{array}{l}\text { The DERMS predicted capacity and reverse-flow violations on the } \\
\text { distribution system and optimally dispatched DERs to correct } \\
\text { these violations. }\end{array}$ \\
\hline $\begin{array}{l}\text { Mitigate voltage issues } \\
\text { with real-power output }\end{array}$ & $\begin{array}{l}\text { The DERMS identified voltage violations on the distribution } \\
\text { system, recommended plans to dispatch real power from DER } \\
\text { aggregations, and optimally dispatched DERs to correct these } \\
\text { violations. }\end{array}$ \\
\hline $\begin{array}{l}\text { Mitigate voltage issues } \\
\text { with reactive power }\end{array}$ & $\begin{array}{l}\text { The DERMS identified voltage violations on the distribution } \\
\text { system, recommended plans to dispatch reactive power from DER } \\
\text { aggregations, and optimally dispatched DERs to correct these } \\
\text { violations (real-time only }) .\end{array}$ \\
\hline $\begin{array}{l}\text { Economic dispatch of } \\
\text { distributed generation } \\
\text { and energy storage }\end{array}$ & $\begin{array}{l}\text { The DERMS created distribution-level signals that could be } \\
\text { integrated into an economic dispatch protocol via an ask-bid- } \\
\text { commit paradigm, for distribution services alone or in combination } \\
\text { with wholesale market participation. }\end{array}$ \\
\hline $\begin{array}{l}\text { Operational flexibility } \\
\text { The DERMS adjusted DER dispatch schedules to real-time } \\
\text { switching and abnormal switching operations. }\end{array}$ \\
\hline
\end{tabular}

\footnotetext{
${ }^{7}$ The real-power use cases included day-ahead and real-time dispatches, while the reactive power use case included real-time dispatches only.
} 
The DERMS enabled BTM and front-of-the-meter batteries to provide distribution grid services while also bidding into the

Enable MUA of DERs wholesale market in a limited set of scenarios. The DERMS used schedules from battery wholesale market participation to optimize distribution activity.

A key objective of the PG\&E DERMS demonstration was to derive a system and user interface that provides greater visibility into real-time and predicted grid conditions by calculating and/or forecasting and visualizing the native load, generation, and net load in real time. Real-time data collection and spatial visualization improve the distribution utility's ability to operate a more dynamic grid with high DER penetration safely and reliably. Figure 7 depicts a snapshot of the PG\&E DERMS demonstration software interface, which includes numerous concurrent data streams such as actual and forecasted load, actual and forecasted DER generation, actual and forecasted system violations, and various DER dispatch-optimization plans (PG\&E 2017).

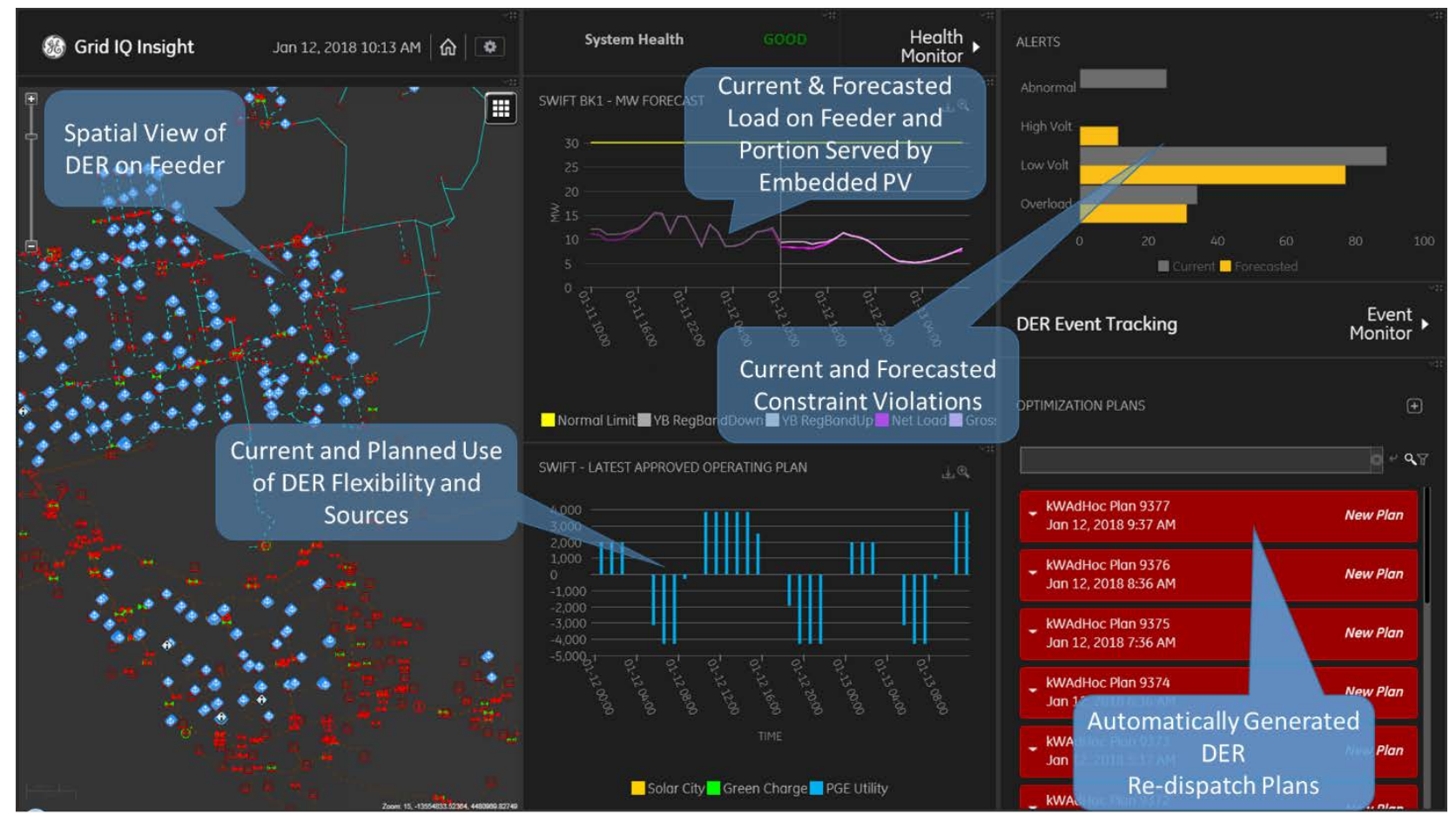

Figure 7. Static illustration of PG\&E DERMS demonstration software interface (PG\&E 2017)

As an example, Figure 8 illustrates the successful operation of the DERMS to mitigate a forecasted distribution system overload condition. The system forecasted a period of relatively low system net load during peak PV hours from 8 a.m. to 6 p.m., followed by a net load spike as waning PV output and increasing power demand caused a projected overload that would have occurred at approximately 8 p.m. The DERMS recognized the overload potential and coordinated DERs to charge during the period of low net load and discharge during high-load times in order to mitigate the potential overload situation that evening. 


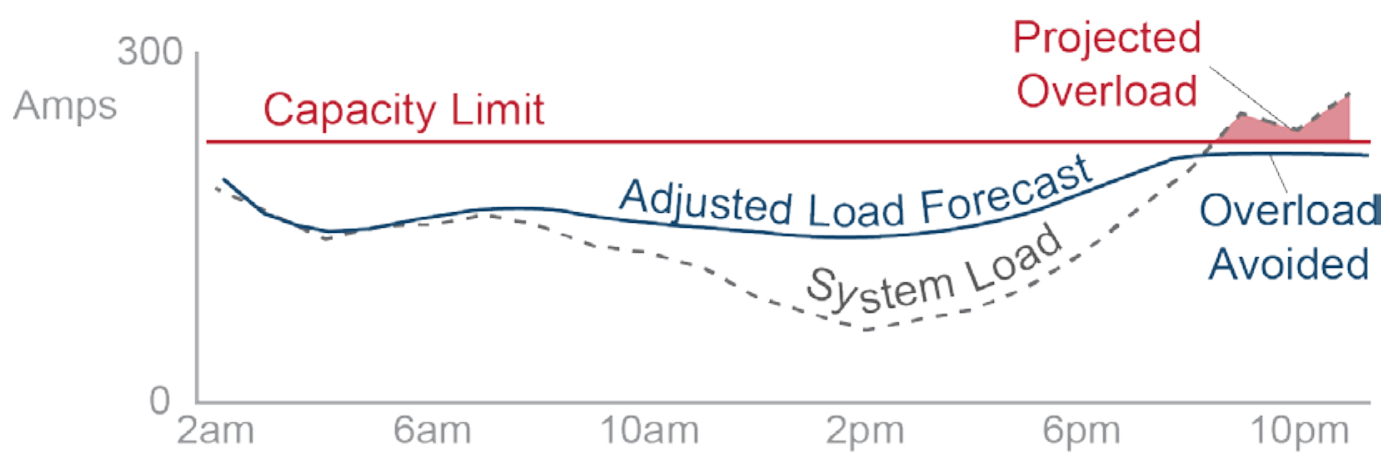

Figure 8. Forecasted DERMS-enabled overload mitigation example

Figure adapted from Portilla (2017)

\subsection{Lessons Learned}

Table 5 summarizes key lessons learned by use case. In addition, PG\&E learned several lessons about DERMS applications more broadly.

PG\&E found that DERs could be aggregated more effectively when multiple assets with shared characteristics could be grouped into aggregation nodes. Aggregation nodes resulted in more efficient exchanges of information between the DERMS and the third-party aggregators. Rather than communicating with individual assets, PG\&E could use the DERMS to communicate with nodes of assets with common characteristics. These characteristics may include spatial and temporal qualities, asset type, and ownership information, among others.

PG\&E discovered that the customer recruitment and DER deployment process was more challenging than anticipated. For future DER deployment targeted to specific locations, considerations for customer-acquisition challenges and timelines will be important - particularly when designing similar DERMS and DER aggregation demonstration projects or when counting on DERs for grid services as part of a grid-deferral non-wires alternative approach.

PG\&E worked with multiple aggregators to better understand differences across providers and in anticipation of a scenario with multiple PV and storage assets in a single area. Working with one aggregation provider might have limited the experience to a single sector (e.g., residential or commercial) while offering only a single perspective of a provider's relative capabilities or limitations. PG\&E also found that working with multiple third-party aggregators represented an opportunity for collaboration, as PG\&E and its partners worked together to clarify the various processes involved and to define requirements and interactions dynamically as the project progressed. However, working with multiple specialized aggregators also added to the complexity of integrating DERs with the DERMS, because such integration is not yet standardized or "plug and play."

Finally, PG\&E helped the DERMS demonstration succeed by introducing each use case in a staged manner, beginning with the least complex and building to the most complex. This sequencing enabled PG\&E to build on the lessons learned at each stage, which enabled identification of points of failure as well as ongoing project reprioritization and redesign. 
Table 5. Lessons Learned by Use Case

\begin{tabular}{ll}
\hline Use Case(s) & Lessons Learned \\
\hline & The demonstration system provided situational awareness \\
& contributing to the system's ability to optimize DERs for various \\
grid needs. This capability is essential to operating the high-DER- & penetration grid and forms the foundation for providing distribution \\
& grid services via DERs. However, PG\&E needed to overcome \\
data quality and availability challenges to enable these functions. & Significant effort was required to improve the data model-which \\
& was incomplete and came from historically disparate systems-by \\
correcting field telemetry issues and overcoming lack of phasing \\
data for the distribution system.
\end{tabular}

DER participation in wholesale markets can affect the accuracy of real-time and forecasted distribution system modeling. In this demonstration, energy storage bidding in wholesale markets complicated the DERMS real-time and forecasted distribution system models. Future DERMS modeling capabilities and/or awareness of DER dispatch schedules will be required to manage this complexity.

DERMS technology can coordinate DERs at test scale to mitigate real-time or forecasted overloads and reverse-power-flow concerns. This project successfully used a DERMS to predict

Manage equipment capacity constraints and reverse power flow capacity and reverse-flow violations on the distribution system, optimize DERs for this constraint, and recommend a plan to dispatch real power from DER aggregations. Both utility and aggregator real-time data and forecasts are critical for effective dispatch. Voltage forecasting was less accurate than power forecasting in the demonstration.

Issuing specific reactive power unit (KVAR) instructions was more effective than issuing power factor setpoint commands because the kVAR instructions provided more precise control over voltage.

Reactive power dispatch addressed voltage issues more effectively than did real-power dispatch. Some real-power dispatch may be necessary in extreme overvoltage situations, although the voltage impact of adjusting real power vs. reactive power depends on specific feeder construction and other location-

Mitigate voltage issues with real/reactive power output dependent factors. kVAR dispatch seemed to have minimal impact on state of charge, and it could be used to provide additional services beyond power (kW) (e.g., provide voltage support if a kW dispatch is causing issues).

DER voltage-control operation should be coordinated with other utility distribution devices. For instance, PG\&E found that DER dispatches to lower voltage could cause other utility distribution devices to increase voltage and cause unwanted operation, resulting in unnecessary wear and tear that could reduce the life expectancy of utility distribution devices. 


\begin{tabular}{ll}
\hline & The least-cost optimization worked well and included energy \\
arbitrage based on market conditions. Although no conclusions \\
can be drawn about the best type of market or valuation of \\
distribution services through this demonstration, PG\&E did identify \\
challenges in implementing a least-cost economic dispatch market \\
structure. In particular, the complexity of the optimization-even at \\
distributed generation \\
and energy storage
\end{tabular}$\quad \begin{aligned} & \text { test scale—created significant computational challenges and, in } \\
& \text { some instances, produced infeasible solutions that required } \\
& \text { manual resolutions. Interactions with the aggregators also } \\
& \text { required customized interfaces to ensure exchange of necessary } \\
& \text { market and technical information. Further exploration of market } \\
& \text { design and distribution-services valuation is necessary. } \\
& \text { The DERMS performed equally well under normal and abnormal } \\
& \text { Switching configurations, although optimally managing DERs to } \\
& \text { provide grid services in abnormal conditions will require the ability } \\
& \text { to dynamically change aggregations of DERs. }\end{aligned}$

\footnotetext{
${ }^{8}$ For example, should the utility reconfigure the distribution system by switching inside of an aggregation node, it can result in an aggregation of DERs being split and served by two different substations. This scenario would necessitate the formulation of new aggregations of DERs and the ability for the utility to dynamically reassign which aggregation a given DER belongs to.
} 


\section{Challenges and Next Steps}

The PG\&E DERMS demonstration provided an encouraging demonstration of core DERMS functions at a test scale. However, several questions and challenges remain to be addressed before DERs can be integrated into utility operational systems at scale.

\section{Scalability}

The DERMS demonstration successfully coordinated $124 \mathrm{~kW}$ of residential PV systems coupled with $66 \mathrm{~kW}(264 \mathrm{kWh})$ of battery storage, $360 \mathrm{~kW}(720 \mathrm{kWh})$ of commercial battery storage, and a 4-MW (28 MWh) PG\&E-owned utility scale battery to provide distribution grid services, with some assets participating in wholesale markets. However, future DERMS may need to coordinate DER fleets with thousands of systems, and much higher capacities, while maintaining safe, reliable, and cost-effective grid operations. PG\&E identified several key areas to address to operate the future high-DER-penetration grid:

- Utility investment in systems and foundational capabilities may be necessary for coordinating DERs. Examples include distribution system planning tools, integrated operational platforms (ADMS, DERMS), enhanced system modeling and analytics, advanced communications, and cybersecurity measures.

- It is very important to consider the coordination of DERs in concert with traditional distribution operations equipment when designing an integrated utility platform. A centralized DERMS platform can help realize new value from DERs while maintaining grid safety and reliability at high DER penetrations.

- Improved communications systems telemetry is necessary to increase visibility into the operational status and availability of DERs for providing distribution services.

- It is useful for utilities to have a scalable and interoperable DER aggregation platform that enables real-time, two-way secure communication between DERs/DER aggregations and distribution utility systems.

\section{Standardization}

Competition between aggregators could drive down DER aggregation costs and spur aggregators to test innovative software platforms that improve usability and DER coordination. At the same time, markets with many aggregators may challenge utilities that are addressing heterogeneous aggregator software, protocols, and standards. Higher levels of standardization may be necessary to ensure that distribution utility requests are interpreted consistently across aggregators, ensure a common understanding of fundamental concepts such as flexibility, and measure key operating metrics to assess aggregator performance. The Institute of Electrical and Electronics Engineers (IEEE) has drafted a standard protocol for utility communication with DERs. However, according to PG\&E, the standard is not yet prescriptive or stable enough to enable consistent interpretation by aggregators for all desired functions.

\section{Targeted DER Deployment}

PG\&E found that demonstration participant recruitment was challenging, which may foreshadow broader challenges related to targeted DER deployment. DERs can provide services only when present in sufficient quantities, at the optimal locations, at the time and duration required by the grid, and when they are more cost-effective than other approaches. Ideally, DERs would be sited 
at critical points where they can provide the greatest value to the grid. However, DER siting may depend on customer acquisition and the interactions of customers with third-party aggregators, which may not yield an optimal site portfolio from a grid perspective.

\section{Valuation}

The DERMS demonstration did not attempt to quantify the value of DER-provided services or the costs of avoided violations. Further valuation research is required to clarify how to value distribution services such as real/reactive power for voltage control and DER use for switching operations.

\section{Market Design}

The DERMS demonstration showed that DERs could be coordinated through an ask-bid-commit market structure, but it did not address compensation, settlement, market design, or market rules issues that are central to the feasibility of any distribution-level market at scale. In particular, future market designs would need to address potential market power accrued by aggregators that have assets at critical points on the grid. For instance, an aggregator with assets on a distribution feeder prone to voltage violations could bid up asking prices if market design leaves the utility dependent on that aggregator to address those violations. The ask-bid-commit market structure may not be viable until key market-design elements are determined. In the near term, simpler dispatch methods, such as direct control or signaling, will likely provide for greater ease of implementation.

\section{Operational Flexibility}

The DERMS demonstration did not attempt to incorporate DERs into optimal switching plans. Future demonstration projects could explore how DERs could be grouped into multiple aggregations, each capable of operating autonomously from the main aggregation, in the case of a switching operation.

\section{Human Capital}

The future of the electric grid may rely increasingly on strategic partnerships between utilities, technology vendors, and aggregators that work toward maximizing the value of DERs to the grid. With the necessary ongoing commitment and investments, projects like the PG\&E DERMS demonstration can play an important role in achieving this goal. 


\section{References}

Barbose, G., and N. Darghouth. 2017. Tracking the Sun 10. Berkeley, CA: Lawrence Berkeley National Laboratory.

Braff, W., J. Mueller, and J. Trancik. 2016. "Value of storage technologies for wind and solar energy." Nature Climate Change 6: 964-969.

CEC and CPUC (California Energy Commission and California Public Utilities Commission). 2015. Recommendations for Utility Communications with Distributed Energy Resources (DER) Systems with Smart Inverters: Smart Inverter Working Group Phase 2 Recommendations. Draft v9, February 28, 2015. Sacramento: CEC.

http://www.energy.ca.gov/electricity analysis/rule21/documents/SIWG Phase 2 Communicatio ns Recommendations for CPUC.pdf.

CPUC (California Public Utilities Commission). 2017. "Research and Development." Accessed September 2017. http://www.cpuc.ca.gov/energyrdd/.

Gartner. 2018. "IT Glossary: Advanced Distribution Management Systems (ADMS).” Accessed August 2018. https://www.gartner.com/it-glossary/advanced-distribution-management-systemsadms.

Mulherkar, A. 2017. North American DER Management Systems 2017-2021. Boston: GTM Research.

PG\&E (Pacific Gas and Electric). 2018. Personal communication.

PG\&E (Pacific Gas and Electric). 2017. Pacific Gas and Electric Company Attachment A. 2016 Annual Report. San Francisco: PG\&E. https://www.pge.com/pge_global/common/pdfs/aboutpge/environment/what-we-are-doing/electric-program-investment-charge/2016-EPIC-Annual$\underline{\text { Report.pdf. }}$

Portilla, A. 2017. "PG\&E DERMS Demonstration Experience." Presented at the Distributech Conference and Exhibition, San Diego, January 31-February 2, 2017. 


\section{Appendix: The Electric Program Investment Charge (EPIC) Program}

The PG\&E DERMS demonstration was funded under California's 2015-2017 EPIC program, the California Public Utilities Commission's (CPUC's) extensive research portfolio for achieving the state's climate and environmental goals. ${ }^{9}$ The EPIC program supports research, demonstration, and deployment projects through 3-year funding cycles, with funding distributed to the California Energy Commission and the state's three investor-owned utilities: PG\&E, Southern California Edison, and San Diego Gas \& Electric. The program enables these utilities to develop emerging technology demonstration and deployment (TD\&D) projects and evaluate how they support safety, reliability, and affordability objectives for the benefit of their customers. Key regulatory drivers to achieve these goals include the following: 1) state energy storage mandates that require PG\&E to operationalize approximately $290 \mathrm{MW}$ of distributionconnected and customer-sited storage by 2024, 2) the CAISO Energy Storage and Aggregated Distributed Energy Resources Initiative for enabling distribution-level energy storage and aggregated DERs to participate in CAISO's transmission-level energy markets, and 3) CPUC requirements under the Distribution Resources Plan for demonstration of multiple DERs as a grid resource.

The main EPIC program areas include applied research and development, TD\&D, and market facilitation (CPUC 2017). The three California investor-owned utilities can participate in EPIC TD\&D projects, which PG\&E categorizes internally into four demonstration program areas: 1) renewable energy and DER integration, 2) grid modernization and optimization, 3) customer service and enablement, and 4) cross-cutting and foundational.

Figure 9 depicts the PG\&E DERMS demonstration (EPIC 2.02 DERMS) within the broader context of the CPUC and PG\&E EPIC program efforts. The DERMS demonstration builds on key data and insights from several other EPIC PG\&E projects, such as EPIC 2.03A Smart Inverters and EPIC 2.19C BTM Storage, which were colocated with the DERMS demonstration.

\footnotetext{
${ }^{9}$ The complete statutory and legislative history of the EPIC program can be found in CPUC (2017).
} 


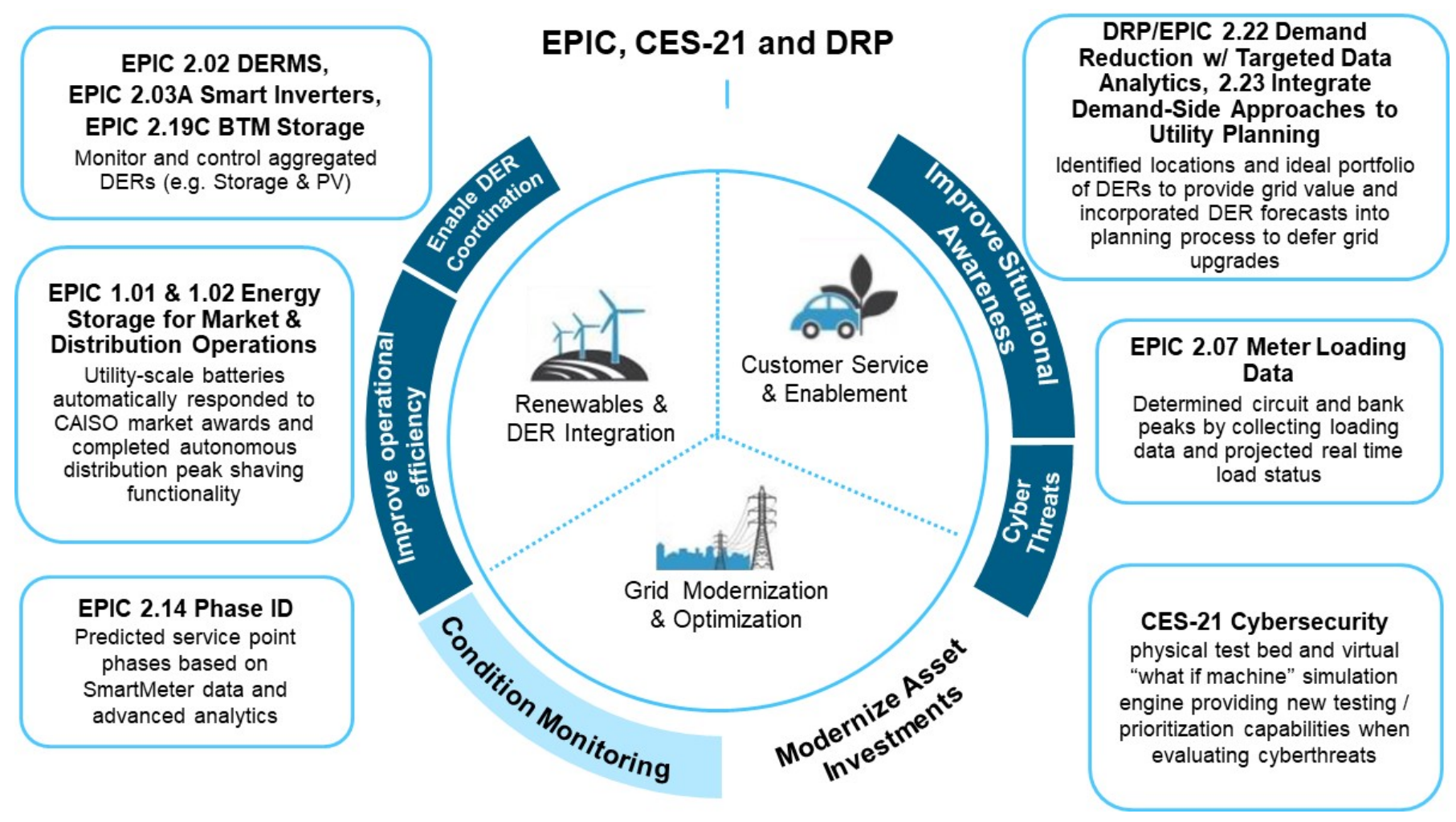

Figure 9. Subset of PG\&E DER-related TD\&D portfolio (PG\&E 2017) 